\title{
Andrographolide alleviates bleomycin-induced NLRP3 inflammasome activation and epithelial-mesenchymal transition in lung epithelial cells by suppressing AKT/mTOR signaling pathway
}

\author{
Jingpei $\mathrm{Li}^{1,2 \#}$, Xiaohan Yang ${ }^{3 \#}$, Penghui Yang ${ }^{1,2}, \mathrm{Ke}_{\mathrm{Xu}}{ }^{1,2}$, Xiaomin Peng ${ }^{1,2}$, Weipeng Cai ${ }^{1,2}$, Simin Zhao ${ }^{1,2}$, \\ Lei Hu ${ }^{4}$, Zhuoyi Li ${ }^{1,2}$, Fei Cui ${ }^{1,2}$, Wei Wang ${ }^{1,2}$, Guilin Peng ${ }^{1,2}$, Xin Xu ${ }^{1,2}$, Jianxing He ${ }^{1,2}$, Jun Liu ${ }^{1,2}$ \\ ${ }^{1}$ Department of Thoracic Surgery, The First Affiliated Hospital of Guangzhou Medical University, Guangzhou, China; ${ }^{2}$ State Key Lab of Respiratory \\ Diseases, National Clinical Research Center for Respiratory Disease, Guangzhou Institute of Respiratory Health, The First Affiliated Hospital of \\ Guangzhou Medical University, Guangzhou, China; ${ }^{3}$ Medical Genetic Centre, Guangdong Women and Children Hospital, Guangzhou, China; \\ ${ }^{4}$ Department of Pharmacy, Peking University People's Hospital, Beijing, China \\ Contributions: (I) Conception and design: All authors; (II) Administrative support: J He, J Liu, J Li; (III) Provision of study materials or patients: X \\ Yang, P Yang, K Xu, W Cai; (IV) Collection and assembly of data: X Peng, W Cai, S Zhao, L Hu, Z Li, F Cui, W Wang, G Peng, Xin X; (V) Data \\ analysis and interpretation: W Wang, L Hu, Z Li, F Cui; (VI) Manuscript writing: All authors; (VII) Final approval of manuscript: All authors. \\ \#These authors contributed equally to this work. \\ Correspondence to: Jianxing He; Jun Liu. Department of Thoracic Surgery, The First Affiliated Hospital of Guangzhou Medical University, No. 151 \\ Yanjiang Road, Guangzhou 510120, China. Email: liujun9707@sina.com; drjianxing.he@gmail.com.
}

Background: Andrographolide (Andro), a diterpenoid extracted from Andrographis paniculata, has been shown to attenuate pulmonary fibrosis in rodents; however, the potential mechanisms remain largely unclear. This study investigated whether and how Andro alleviates bleomycin (BLM)-induced NOD-like receptor family pyrin domain containing 3 (NLRP3) inflammasome activation and epithelial-mesenchymal transition (EMT) in the lung epithelial cells.

Methods: The in vivo effects of Andro were evaluated in a rat model of BLM-induced pulmonary fibrosis. The roles of Andro in BLM-induced NLRP3 inflammasome activation, EMT and AKT/mTOR signaling were investigated using human alveolar epithelial A549 cells.

Results: We found that Andro significantly alleviated pulmonary edema and histopathological changes, decreased weight loss, and reduced collagen deposition. Andro downregulated the levels of NLRP3, the adaptor molecule apoptosis-associated speck-like protein containing a CARD (ASC), and Caspase-1 in the lungs of BLM-treated rats, suggesting the inhibitory effect of Andro on NLRP3 inflammasome activation in vivo. Additionally, the symptoms of BLM-mediated EMT phenotype in the lung were also attenuated after Andro administration. In vitro, Andro also markedly inhibited BLM-induced NLRP3 inflammasome activation and EMT in A549 cells. Moreover, Andro inhibited BLM-induced phosphorylation of AKT and mTOR in A549 cells, suggesting that AKT/mTOR inactivation mediates Andro-induced effects on BLMinduced NLRP3 inflammasome activation and EMT.

Conclusions: These data indicate that Andro can reduce BLM-induced pulmonary fibrosis through suppressing NLRP3 inflammasome activation and EMT in lung epithelial cells via AKT/mTOR signaling pathway.

Keywords: Andrographolide (Andro); bleomycin (BLM); NOD-like receptor family pyrin domain containing 3 inflammasome (NLRP3 inflammasome); epithelial-mesenchymal transition (EMT); AKT/mTOR signaling

Submitted Dec 14, 2020. Accepted for publication Mar 12, 2021.

doi: $10.21037 /$ atm-20-7973

View this article at: http://dx.doi.org/10.21037/atm-20-7973 


\section{Introduction}

Idiopathic pulmonary fibrosis (IPF) is a devastating and untreatable lung disease of unknown etiology $(1,2)$. It is characterized by abundant deposition of extracellular matrix $(\mathrm{ECM})$ in the lung $(3,4)$. Increasing ECM generation alters the lung architecture and leads to subsequent respiratory dysfunction, ultimately resulting in respiratory failure. Eventually, patients need lung transplantation to subsist. There is no cure for IPF, and its emergence across the world gradually increases (5). Hence, comprehending the underlying molecular mechanisms is very important for identifying and developing an effective treatment for IPF.

Bleomycin (BLM), a commonly-used antineoplastic agent, induces the interstitial pulmonary fibrosis in a dosedependent manner (6). Intratracheally administered BLM into the rodent lungs triggers lung epithelial cell damage, inflammation, epithelial-mesenchymal transition (EMT) and ECM production, which resembles human IPF $(4,7)$. Thus, BLM-induced pulmonary fibrosis is still the most widely-used model for IPF (8). Alveolar epithelial cells (AECs) are thought of as a critical target of BLM lung toxicity (9). Exposure of cells to BLM also causes diverse cellular responses, such as NOD-like receptor family pyrin domain containing 3 (NLRP3) inflammasome activation and EMT, which result in the development of lung fibrosis induced by BLM (10).

AECs can procure a mesenchymal phenotype by way of the EMT after lung injury, which is known as an important source for activated fibroblasts in IPF $(11,12)$. In recent years, more and more researchers have been immersed in the effects of EMT in pulmonary fibrosis $(13,14)$. In experimental pulmonary fibrosis in mice, about one third of fibroblasts were derived from AECs two weeks after BLM administration (15). It has been reported that BLM can induce EMT in AEC lines (for example, A549 and RLE-6TN cells). Therefore, inhibition of the EMT process is helpful for the attenuation of BLMinduced pulmonary fibrosis.

Inflammasomes are multiprotein complexes that promote maturation and secretion of proinflammatory cytokines such as interleukin (IL)- $1 \beta$ and IL-18 via caspase- 1 activation (16). There are five types of inflammasomes: NLRP1, NLRP3, NLRC4, IPAF, and AIM2 inflammasomes respectively. Inflammasome activation is also considered to play an important role, since both NLRP3 and AIM2 inflammasome activation can contribute to lung fibrogenesis $(10,16,17)$. The NLRP3 inflammasome, a typical member of the inflammasome family, consists of the NLRP3, the adaptor molecule apoptosis-associated speck-like protein containing a CARD (ASC), and the effector molecule procaspase-1. Its activation promotes the maturation of caspase-1, and accelerates the inflammatory cytokine production, for instance, IL-1 $\beta$ and IL-18 (18). Increasing researchers have demonstrated that the levels of NLRP3, caspase-1, and IL$1 \beta$ were significantly increased in IPF patients (19). NLRP3 inflammasome activation is also observed in asbestos-, silicaand BLM-induced pulmonary fibrosis (20). In NLRP3deficient mice, a significant decrease was observed in BLMinduced pulmonary fibrosis (16). Hence, inhibition of NLRP3 inflammasome activation can alleviate pulmonary fibrosis (10).

Andrographolide (Andro), a bicyclic diterpenoid lactone extracted and purified from Andrographis paniculata, exerts antioxidant, anti-inflammatory, and immunomodulatory properties $(21,22)$. Recently, Andro has been shown to inhibit the cigarette smoke-induced EMT by inhibiting HOTAIR (23). Andro not only attenuated OVA-induced NLRP3 inflammasome activation in mice, but also suppressed NLRP3 inflammasome activation in the in-vitro and in-vivo models of Parkinson disease (24). We and other teams have found that Andro was able to inhibit BLMinduced pulmonary fibrosis in mice (25-27). Karkale et al. have previously found that Andro inhibited silica-induced EMT phenotype in the lungs (28). Gao et al. found that Andro also reduced AIM2 inflammasome-mediated pyroptosis in radiation-induced lung fibrosis (29). Our research shows that Andro also suppressed TGF- $\beta 1$ induced EMT in A549 cells (30). However, as far as we know, it remains unclear on whether Andro directly affects the BLM-induced NLRP3 inflammasome activation and EMT phenotype in AECs.

The aim of this study was to investigate the impact of Andro on BLM-induced NLRP3 inflammasome activation and EMT in the AECs in vivo and in vitro, and explore the underlying mechanism of action. Our results showed that Andro directly attenuates BLM-induced NLRP3 inflammasome activation and EMT in AECs through AKT/mTOR signaling pathway. We present the following article in accordance with the ARRIVE reporting checklist (available at http://dx.doi.org/10.21037/atm-20-7973).

\section{Methods}

\section{Reagents}

Andro was provided by Chengdu Herbpurify Co., Ltd. 
(Chengdu, China). BLM was provided by Hisun Pfizer Pharmaceuticals Co., Ltd. (Hangzhou, China). Antibodies to E-cadherin, ASC, cleaved caspase 1, p-mTOR, mTOR, p-AKT, and AKT were bought from Cell Signaling Technology (CA, USA). Antibodies to fibronectin, NLRP3, and GAPDH were bought from ABclonal (Wuhan, China). Antibody to alpha-smooth muscle actin ( $\alpha$-SMA) were bought from Sigma-Aldrich (St Louis, MO, USA). Antibodies to the HRP-labeled Goat Anti-Rabbit/Mouse $\operatorname{IgG}(\mathrm{H}+\mathrm{L})$ were bought from Abcam Biotechnology (Cambridge, MA, USA).

\section{Animals and treatments}

Six to 8 week-adult male Sprague-Dawley rats $(200 \pm 10 \mathrm{~g})$ were obtained from Guangdong Medical Experimental Animal Center (Guangzhou, Guangdong, China), and reared in a special pathogen-free room, where they were allowed to gain food and water ad libitum. Experiments were performed under a project license (No. 2017-346) granted by the Animal Care and Use Committee of the First Affiliated Hospital of Guangzhou Medical University, in compliance with institutional guidelines for the care and use of animals. The rats were divided into four groups: saline $+0.5 \%$ sodium carboxymethyl cellulose (CMC-Na) (Con group), saline + Andro (10 mg/kg, Andro group), BLM (3.0 mg/kg) + 0.5\% CMC-Na (BLM group), and BLM + Andro (BLM + Andro group). Saline or BLM solution was intratracheally injected to the rats on day 0 . On the following day, intragastrically administered 0.5\% CMC-Na or Andro was made daily for 21 days. After the rats were sacrificed, the lungs were harvested for the analysis described below. There were at least 5 mice per group in each animal experiment.

\section{Masson's trichrome staining}

Left lungs were fixed in 4\% paraformaldehyde, embedded in paraffin and then cut into $5 \mu \mathrm{m}$-thick slices. Whereafter, Masson trichrome staining was performed using a kit (Sigma-Aldrich, USA) according to the manufacturer's instruction. Histopathological scores of fibrosis were assessed according to the descriptions by Ashcroft (31). The degree of interstitial fibrosis was graded using a scale of 0 to 8 as follows: grade 0 , normal lung; grade 1, minimal fibrous thickening of alveolar or bronchial walls; grade 2-3, moderate thickening of walls without obvious damage to lung architecture; grade 4-5, increased fibrosis with definite damage to lung architecture and formation of fibrous bands or small fibrous mass; grade 6-7, severe distortion of structure and large fibrous areas; "honeycomb lung" was included in this category; grade 8 , total fibrous obliteration of the field.

\section{Immunohistochemistry}

Immunohistochemistry was performed using E-cadherin (1:100) or $\alpha$-SMA (1:200) antibodies as described previously (27).

\section{Real-time PCR}

Total RNA was extracted from lung tissues using TRIzol Reagent (Invitrogen Corporation, CA, USA) and reversetranscribed into first-strand cDNA using the PrimeScript RT reagent Kit with gDNA Eraser (Takara, Shiga, Japan). The mRNA levels of collagen 1, collagen 3, E-cadherin, fibronectin, $\alpha$-SMA, NLRP3, ASC, and Caspase 1 were examined by Real-time PCR using SYBR Green Master Mix Kit (Yeasen Biotech Co., Ltd., Shanghai, China). The primer sequences for real-time PCR assays are listed in Table 1.

\section{Cell culture}

Human alveolar epithelial A549 cells were bought from Cell Bank of the Chinese Academy of Sciences (Shanghai, China), and were grown in DMEM including $10 \%$ FBS, $100 \mathrm{KU} / \mathrm{L}$ penicillin and $100 \mathrm{mg} / \mathrm{L}$ streptomycin in a $37^{\circ} \mathrm{C}$ humidified incubator with $5 \% \mathrm{CO}_{2}$. Each cell experiment was at least repeated three times.

\section{Western blot}

Western blot was performed as previously described (27). The immunoreactive bands were scanned using the Tanon-5200 (Tanon Science \& Technology Co., Ltd., Shanghai, China). The intensity analysis was analyzed by Image J. The used primary antibodies were as follows: anti-E-cadherin antibody $(1: 1,000)$, anti-Fibronectin antibody $(1: 1,000)$, anti- $\alpha$-SMA antibody (1:3,000), anti-p-AKT antibody (1:1,000), anti-AKT antibody $(1: 1,000)$, anti-p-mTOR antibody $(1: 1,000)$, antimTOR antibody $(1: 1,000)$, anti-NLRP3 antibody $(1: 1,000)$, anti-cleaved caspase 1 antibody $(1: 1,000)$, anti-ASC antibody $(1: 1,000)$, and rabbit anti-GAPDH antibody $(1: 3,000)$.

\section{Statistical analysis}

Data analysis was performed using SigmaPlot 12. 5 and 
Table 1 Primers used for real-time PCR

\begin{tabular}{lll}
\hline Gene & Sense primer $\left(5^{\prime}-3^{\prime}\right)$ & Antisense primer $\left(5^{\prime}-3^{\prime}\right)$ \\
\hline Collagen $1(r)$ & ATCCTGCCGATGTCGCTAT & CCACAAGCGTGCTGTAGGT \\
Collagen $3(r)$ & CTGGTCCTGTTGGTCCATCT & ACCTTTGTCACCTCGTGGAC \\
E-cadherin $(r)$ & GGCCCAGGAGCTGACAAAC & CCAGAGGCTGCGTCACTTC \\
Fibronectin $(r)$ & CATAGCAGGTACAAACCAGGG \\
$\alpha$-SMA $(r)$ & TCCAGAGTCCAGCACAATACCAG & AATGACCCAGATTATGTTTGAGACC \\
NLRP3 $(r)$ & CAGACCTCCAAGACCACGACTG & TTTAATGTCACGCACGATTC \\
ASC $(r)$ & TTATGGAAGAGTCTGGAGCTGTGG & CATCCGCAGCCAATGAACAGAG \\
Caspase $1(r)$ & TGCCTGGTCTTGTGACTTGGAG & ATGTCCTGGGAAGAGGTAGAAACG \\
GAPDH $(r)$ & AAGGTGGTGAAGCAGGCGGC & GAGCAATGCCAGCCCCAGCA \\
\hline
\end{tabular}

presented as means \pm standard error (SE). Differences between multiple groups were analyzed by One-way ANOVA. $\mathrm{P}<0.05$ was considered statistically significant.

\section{Results}

\section{Andro ameliorates BLM-induced pulmonary fibrosis in rats}

After BLM injury, an apparent weight loss and an augment in lung wet-to-dry weight ratio were observed (Figure 1A,B). However, these changes were attenuated by Andro treatment. Masson's trichrome staining showed remarkable collapse of alveolar septa, loss of lung architecture and excess deposition of collagen in BLM-treated rats comparing with control group. Whereas treatment with Andro decreased the injury to lung architecture and the deposition of collagen owing to BLM (Figure 1C). Ashcroft scoring to quantify morphologic fibrosis was performed, and the pathology score in Androtreated rats was significantly decreased relative to the BLM alone treated rats (Figure 1D). In addition, the decline of fibrosis in Andro-treated rats was further demonstrated by downregulation of collagen 1 and collagen 3 levels (Figure 1E,F,G). Collectively, these results suggest Andro ameliorates BLM-induced pulmonary fibrosis in vivo.

\section{Andro represses BLM-induced EMT in pulmonary fibrosis in rats}

The effects of Andro on the BLM-induced EMT in the lungs were investigated. Our immunohistochemistry study showed that BLM down regulated E-cadherin-positive cells and up regulated $\alpha$-SMA-positive cells compared with controls. In Andro-treated rats, the decrease of E-cadherin-positive cells was significantly prevented and the accumulation of $\alpha$-SMApositive cells were reduced (Figure $2 A, B$ ). Additionally, Realtime PCR results indicated that E-cadherin mRNA level was enhanced, whereas fibronectin and $\alpha$-SMA expressions were diminished by Andro treatment (Figure 2C,D,E). Moreover, E-cadherin protein level was enhanced, whereas $\alpha$-SMA protein expression was diminished by Andro treatment (Figure 2F,G,H). Taken together, these findings imply that Andro inhibits the BLM-induced EMT in vivo.

\section{Andro reduces BLM-induced NLRP3 inflammasome activation in pulmonary fibrosis in rats}

As NLRP3 participates in the occurrence of EMT in BLM-induced pulmonary fibrosis. Role of Andro in BLMinduced NLRP3 inflammasome activation in lungs were therefore investigated. As shown in Figure 3, the mRNA levels of NLRP3, ASC, and Caspase-1 and the protein level of NLRP3 were both remarkably upregulated in BLMtreated rat lungs, suggesting the activation of NLRP3 inflammasome in BLM-induced pulmonary fibrosis in vivo. Treatment of Andro attenuated the BLM-induced increase of NLRP3, ASC and Caspase-1 in the lungs. Therefore, Andro reduces BLM-induced NLRP3 inflammasome activation in the lungs.

\section{Andro suppresses BLM-induced EMT in alveolar epithelial A549 cells}

Since AECs, in particular type II AECs, have emerged as 
A

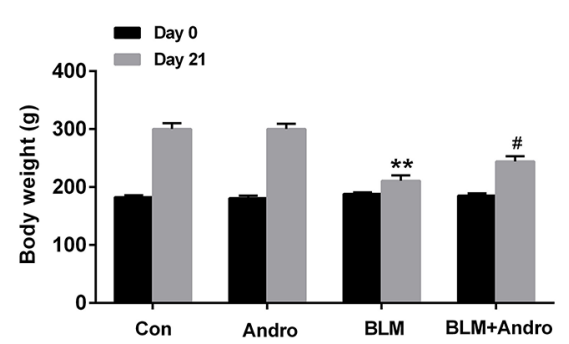

B

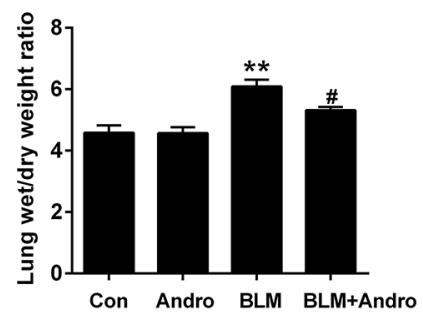

C

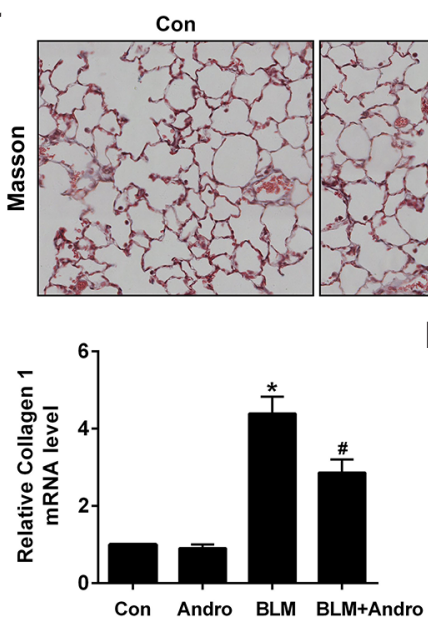

Andro

BLM
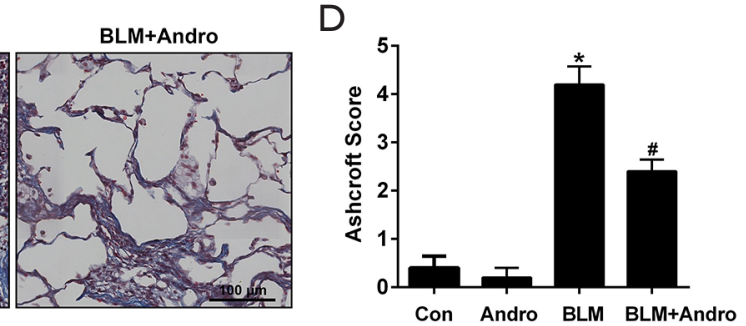

$\mathrm{F}$

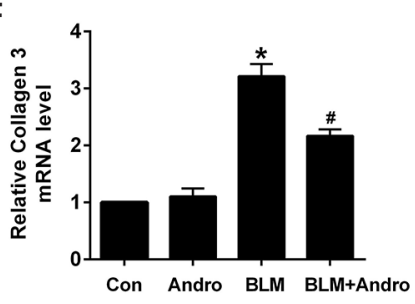

G
Con Andro BLM BLM+Andro

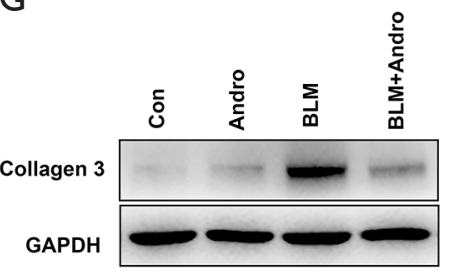

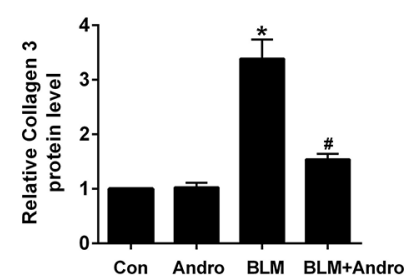

Figure 1 Andro ameliorated BLM-induced pulmonary fibrosis in the lungs. (A) Rat' average weight in each group was compared both on day 0 and on day 21 after BLM administration. (B) Lung wet-to-dry weight ratio in each group. (C) 21 days after BLM instillation, pulmonary pathology was measured by Masson's trichrome staining (scale bar: $100 \mu \mathrm{m}$ ). (D) Pathological grading of fibrosis was performed using the Ashcroft score system. (E,F) The mRNA levels of collagen 1 and collagen 3 were examined by Real-time PCR. (G) The protein level of collagen 3 was examined by Western blot. *, $\mathrm{P}<0.05$ vs. Con group; ${ }^{*}, \mathrm{P}<0.05$ vs. BLM group.

key players in this disease (32), we further assessed the role of Andro in BLM-induced EMT occurrence in the AECs. Resembling the in vivo results, treatment of Andro inhibited BLM-induced EMT in vitro, as demonstrated by the alteration expressions of EMT related protein markers (Figure 4).

\section{Andro suppresses BLM-induced NLRP3 inflammasome activation in alveolar epithelial A549 cells}

We therefore observed the effects of Andro on BLMinduced NLRP3 inflammasome activation in the AECs. As shown in Figure 5, BLM significantly elevated the protein expressions of NLRP3, ASC, and cleaved caspase 1, which were all decreased by Andro treatment, implying the inhibitory effects of Andro on BLM-induced NLRP3 inflammasome activation in the AECs.

\section{Andro suppresses BLM-induced activation of AKT/mTOR signaling pathway in alveolar epithelial A549 cells}

Having established the effectiveness of Andro in BLMinduced NLRP3 inflammasome activation and EMT in AECs, we next inquired into the underlying mechanisms. The involvement of AKT/mTOR signaling in BLM-induced pulmonary fibrosis is well-documented (33). AKT/mTOR signaling is considered as an important mechanism implicated in the process of NLRP3 inflammasome activation and EMT during the pulmonary fibrosis (33). Therefore, we explored whether Andro inhibited BLM-induced NLRP3 inflammasome activation and EMT through inactivation of 
A

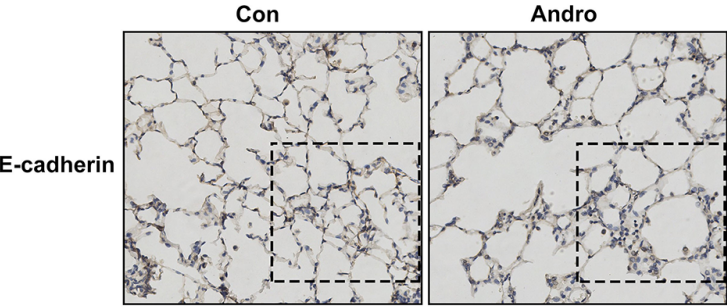

BLM

BLM+Andro
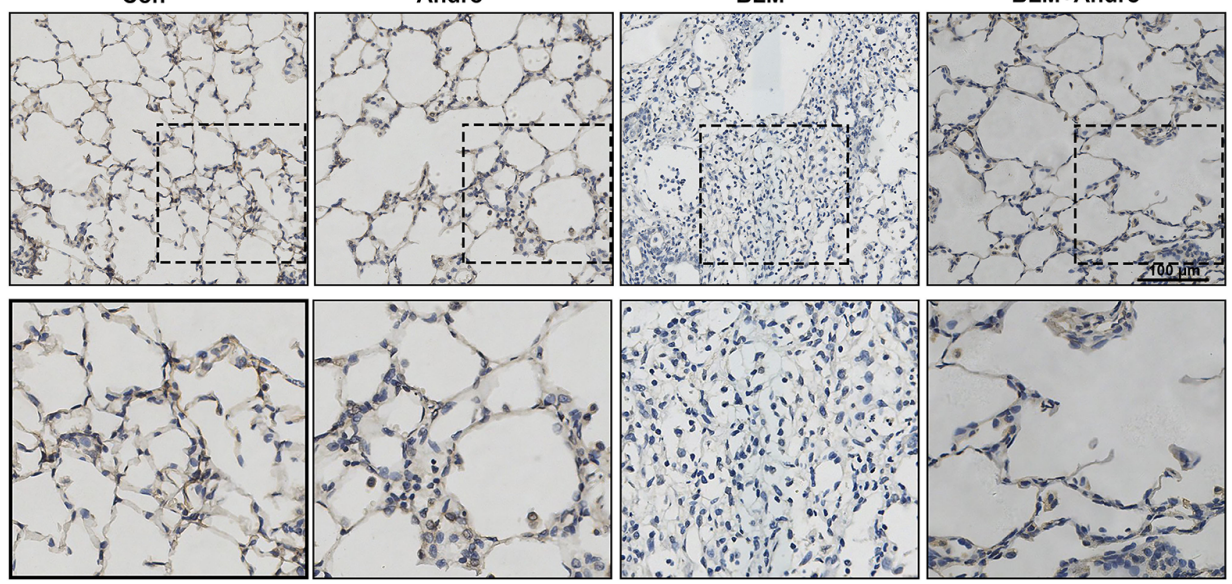

B

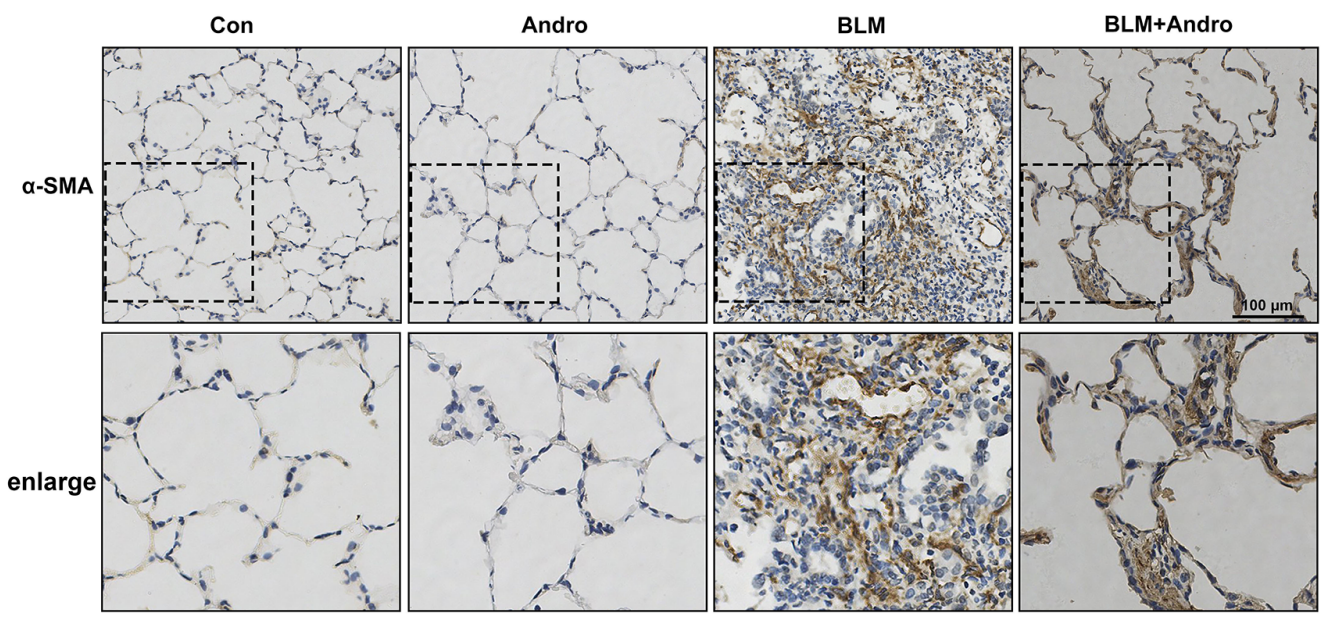

C

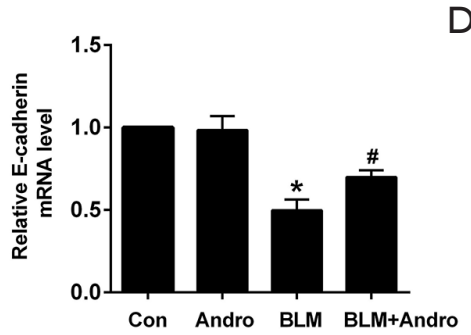

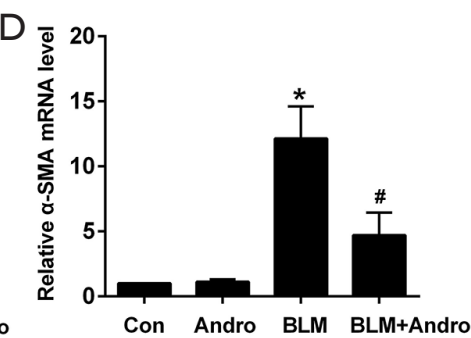

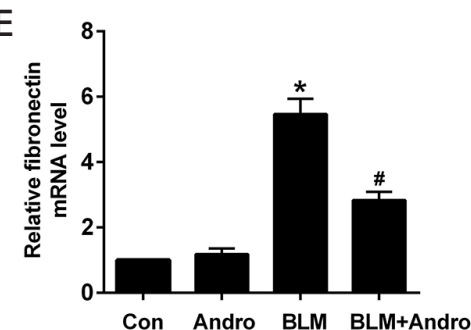

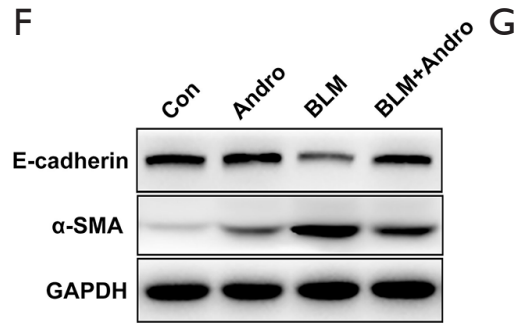
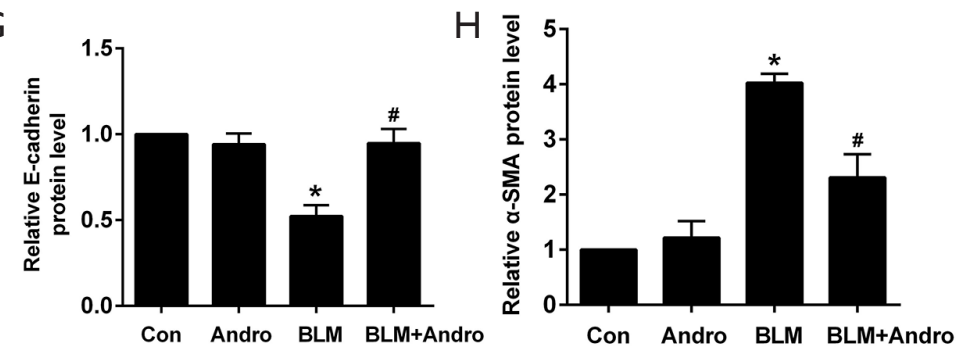

Figure 2 Andro inhibited BLM-induced EMT in the lungs. Twenty-one days after BLM instillation, rats are sacrificed and lungs were collected. (A,B) The positive cells of E-cadherin and $\alpha$-SMA in the lungs were determined by immunohistochemistry assay $(\times 400)$. $(\mathrm{C}, \mathrm{D}, \mathrm{E})$ The mRNA levels of E-cadherin, $\alpha$-SMA and fibronectin were measured by Real-time PCR. (F,G,H) The protein levels of E-cadherin and $\alpha$-SMA were examined by Western blot. *, $\mathrm{P}<0.05$ vs. Con group; ${ }^{*}, \mathrm{P}<0.05$ vs. BLM group. 
A

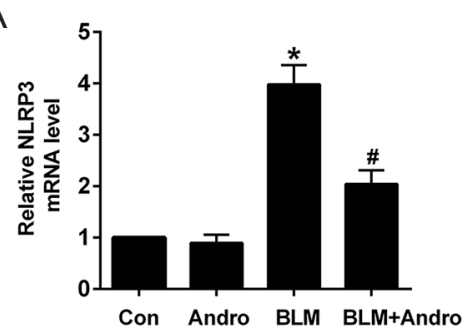

D

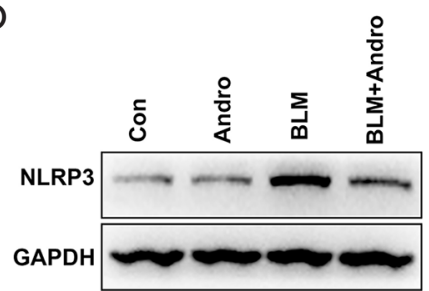

B
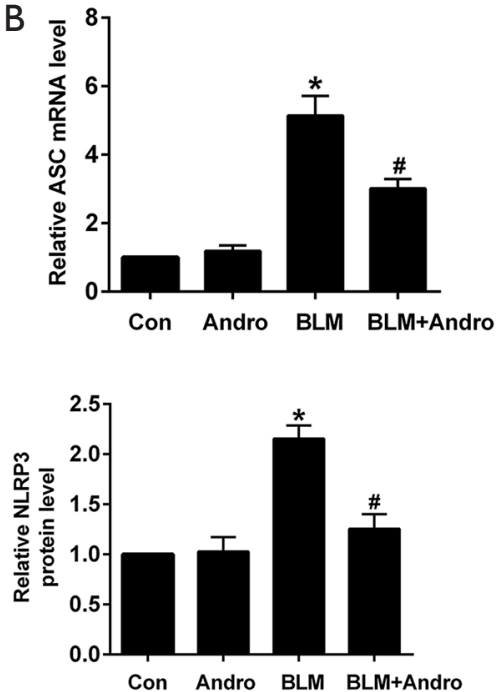

C

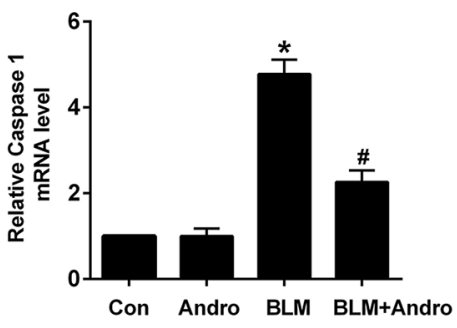

Figure 3 Andro reduced BLM-induced NLRP3 inflammasome activation in the lungs. 21 days after BLM instillation, rats are sacrificed and lungs were collected. Real-time PCR was performed to examine the mRNA levels of NLRP3 (A), ASC (B), and Caspase-1 (C). (D) The protein level of NLRP3 was examined by Western blot. ${ }^{*}, \mathrm{P}<0.05$ vs. Con group; ${ }^{*}, \mathrm{P}<0.05$ vs. BLM group.

A

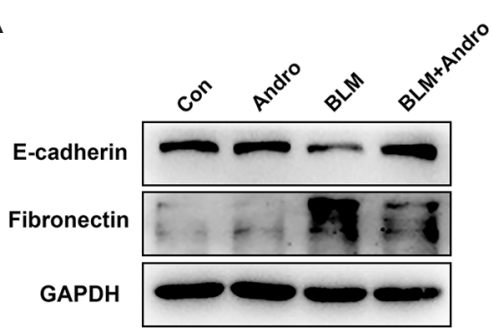

B

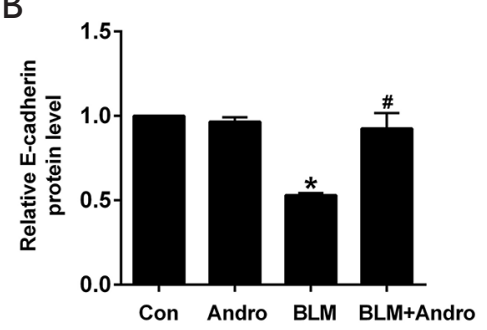

C

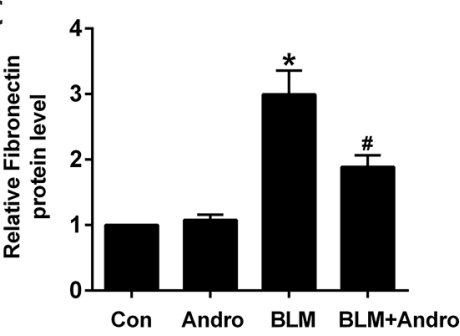

Figure 4 Andro suppressed BLM-induced EMT in human alveolar epithelial A549 cells. A549 cells were treated with BLM and/or Andro for 48 h. (A) The protein expression levels of E-cadherin, and fibronectin were analyzed by Western blot. (B,C) Densitometric analysis of relevant proteins in the immunoblots using GAPDH as the internal reference. *, $\mathrm{P}<0.05$ vs. Con group; ${ }^{*}, \mathrm{P}<0.05$ vs. BLM group.

AKT/mTOR signaling pathway. As shown in Figure 6, we determined the relative expression of $\mathrm{p}$-AKT and $\mathrm{p}$-mTOR by Western blot. The data showed that the protein expression levels of p-AKT and p-mTOR were both significantly increased in the BLM-exposed A549 cells, suggesting the activation of AKT/mTOR signaling pathway in AECs. While treatment with Andro inhibited the BLM-induced activation of AKT $/ \mathrm{mTOR}$ signaling pathway.

\section{Discussion}

In the present study, we further confirmed the phylactic power of Andro on BLM-induced pulmonary fibrosis. Our results, performed in AECs both in the in vitro and in vivo models of BLM-induced pulmonary fibrosis, provided convincing evidence of the critical role of BLM-activated NLRP3 inflammasome activation and EMT, promoting myofibroblast formation and collagen synthesis. The results showed that Andro significantly suppressed BLMinduced NLRP3 inflammasome activation in vivo and in vitro, as indicated by reduced levels of NLRP3, ASC, and cleaved caspase- 1 . We also established that Andro treatment significantly attenuated BLM-induced downregulation of epithelial markers and upregulation of mesenchymal markers in vivo and in vitro, suggesting that Andro also targeted EMT to suppress the formation of myofibroblasts. In this study, 

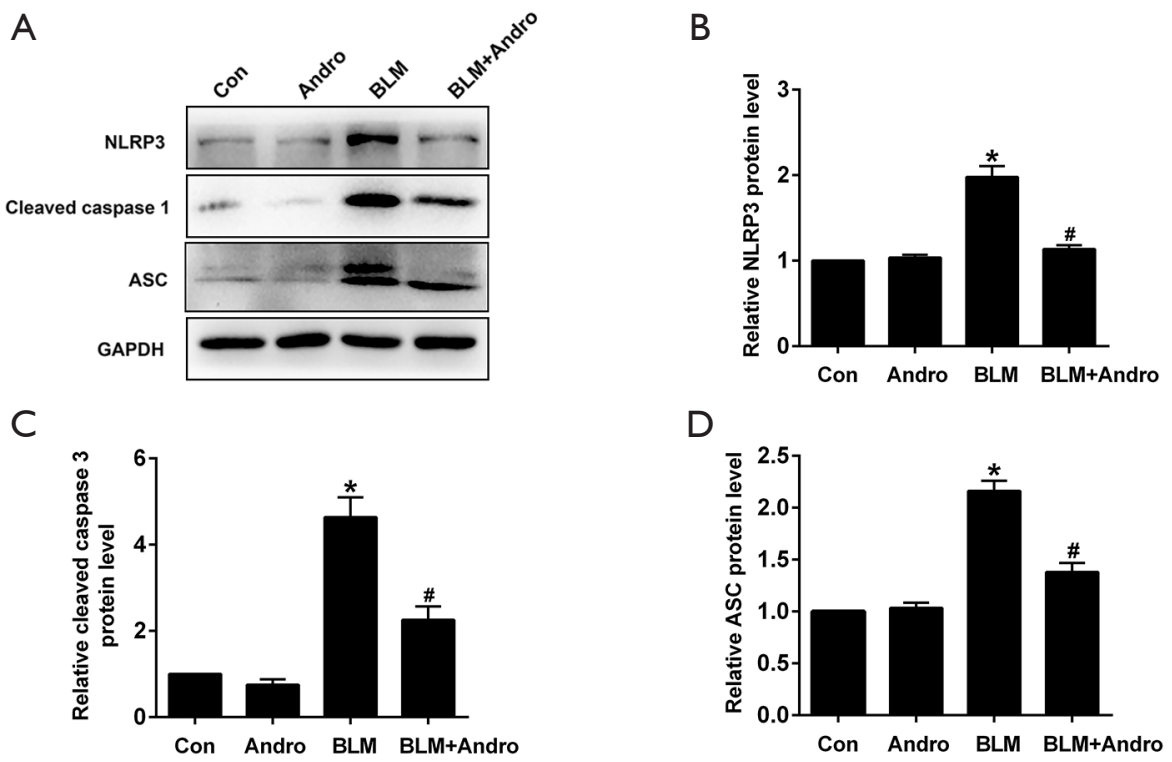

Figure 5 Andro suppressed BLM-induced NLRP3 inflammasome activation in human alveolar epithelial A549 cells. A549 cells were treated with BLM and/or Andro for 48 h. (A) The protein levels of NLRP3, ASC, and cleaved caspase 1 were analyzed by Western blot. (B,C,D) Densitometric analysis of relevant proteins in the immunoblots using GAPDH as the internal reference. * $\mathrm{P}<0.05$ vs. Con group; ${ }^{\#}, \mathrm{P}<0.05$ vs. BLM group.

A

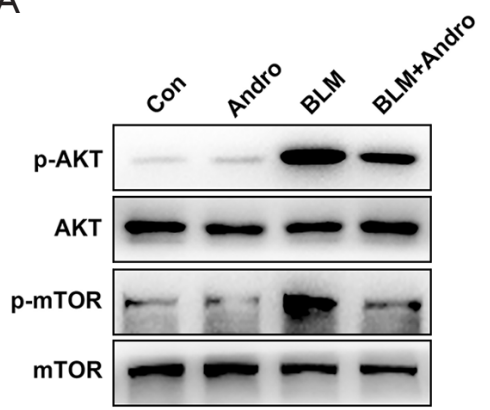

B

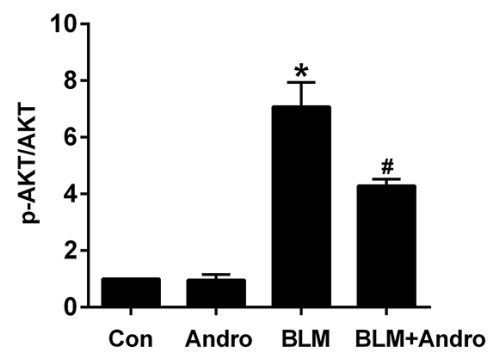

C

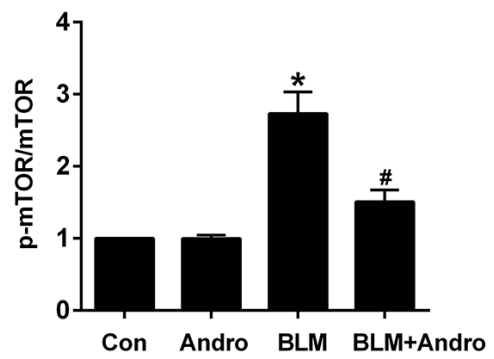

Figure 6 Andro suppressed BLM-activated AKT/mTOR signaling pathway in A549 cells. A549 cells were treated with BLM and/or Andro for 48 h. (A) Western blot analysis was performed to measure the phosphorylation levels of AKT and mTOR. (B,C) Scanning densitometry of western blot on different samples was analyzed quantitatively. Expression of p-AKT and p-mTOR was normalized to AKT and mTOR levels, respectively. *, $\mathrm{P}<0.05$ vs. Con group; ${ }^{*} \mathrm{P}<0.05$ vs. BLM group.

we identified that Andro counteracted BLM-activated AKT/ mTOR signaling pathway to repress NLRP3 inflammasome activation and EMT in AECs, leading to attenuation of BLM-induced pulmonary fibrosis.

IPF is characterized by excessive production and deposition of myofibroblasts and ECM components in the lung that damage its architecture and impairs its function $(32,34)$. Although the pathogenic mechanism of
IPF is largely unknown, lung epithelial cells, type II AECs particularly, have also been considered to be an important player in this disease (34). Accumulating evidence shows that recurrent injury to the AECs is the "prime mover" of pulmonary fibrosis. After injury, the surviving epithelial cells in the lungs with IPF demonstrate an altered phenotype via EMT (35). EMT, a dynamic cellular program, allows polarized epithelial cells to transform into uncontrolled 
mesenchymal cells. In addition to the critical role that EMT has in tumor metastasis, increasing evidence suggests that EMT is also a process involved in fibrotic diseases, and a main source of fibroblasts and myofibroblasts in aggressive pulmonary fibrosis $(1,36)$. A previous study showed that $\sim 33 \%$ of myofibroblasts in the lungs of an experimental BLM fibrosis model were traceable to cells undergoing EMT (15). Given this, inhibition of EMT is also an important way to attenuate pulmonary fibrosis. In the recent decades, there are some reports demonstrating that, besides TGF- $\beta 1$, BLM also directly induces EMT in primary AECs as well as in A549 cells (10). In accordance with this, our results showed that BLM successfully triggered EMT in A549 cells, and treatment with Andro dramatically reduced BLM-induced EMT, which was indicated by alteration of EMT-related biomarkers. We further identified that the surviving epithelial cells in the lungs with BLM-induced pulmonary fibrosis showed an altered phenotype via EMT, as evidenced by decreased epithelial markers and increased mesenchymal cell markers. While treatment with Andro reduced the occurrence of EMT phenotype following BLM stimulation, which is in accordance with previous studies demonstrating that Andro ameliorated silica or cigarette smoke induced EMT in mice $(23,28)$. Research has reported that even the same drug, gene or protein can project diverse effect on different animal models of the same disease. For instance, the receptor for advanced glycation end-products (RAGE) KO mice had a severe fibrosis score, when compared to WT silica-treated mice (37), but were largely protected from the fibrotic effects of BLM (38). Fortunately, Andro not only suppressed silica-induced EMT in mice, but also inhibited BLM-induced EMT in mice. This is very important for the prevention and treatment of different types of patients with pulmonary fibrosis.

In addition, the NLRP3 inflammasome has been found to be activated and plays a key role in numerous respiratory diseases including IPF (10). Increasing evidence has suggested that the NLRP3 inflammasome activation may promote pulmonary fibrosis through amplifying the pro-inflammatory response cascade. Moreover, it has been reported that NLRP3 inflammasome activation also takes part in the modulation of EMT in BLM-induced pulmonary fibrosis (10). Thus, suppressing the activation of the NLRP3 inflammasome can attenuate pulmonary inflammation and fibrosis (20). Our results demonstrated that Andro decreased BLM-induced NLRP3 inflammasome activation in vivo and in vitro, which is in line with previous findings that Andro attenuated NLRP3 inflammasome activation in both experimental non-alcoholic steatohepatitis and OVA-induced lung injury in mice $(24,39)$. This is also in keeping with the finding that Andro repressed NLRP3 inflammasome activation in microglia in in vitro and in vivo models of Parkinson disease (40). Additionally, previous studies have reported that the enhancement of inflammatory response in the lungs after BLM was significantly inhibited by Andro treatment (25), indicating that NLRP3 inflammasome inactivation by Andro may be the reason for attenuation of pro-inflammatory response cascade.

The mechanism by which Andro inhibits NLRP3 inflammasome activation and EMT in the AECs remains obscure. Activation of several signaling pathways including AKT/mTOR signaling, can promote NLRP3 inflammasome activation and EMT process. For instance, studies have shown that AKT/mTOR pathway can regulate NLRP3 activation and inflammation (41). And, the AKT/ mTOR signaling is also essential for EMT, which eventually contributes to the pathobiology of fibrosis (42). Moreover, increasing evidence indicates that AKT/mTOR signaling is significantly activated and plays a crucial role in the development of pulmonary fibrosis (32). Here, we found that the protein expressions of $\mathrm{p}-\mathrm{AKT}$ and $\mathrm{p}-\mathrm{mTOR}$ were significantly increased in BLM-induced mice and decreased after Andro treatment, indicating the Andro's inhibitory effects on NLRP3 inflammasome activation and EMT are associated with the suppression of BLM-induced AKT and mTOR phosphorylation. AKT and mTOR are critical components in a variety of processes which regulate the EMT through suppression of the expression of E-cadherin via EMT transcription factors (43). Our previous study has demonstrated that Snail and Slug, two important EMTrelated transcription factors, were significantly inhibited by Andro, suggesting the inhibitory role of Andro on EMTrelated transcription factors. Therefore, we speculate that the inhibition of EMT by Andro was due to the inhibition of AKT/mTOR signaling via the Snail family members (Snail and Slug). On the basis of these challenging data generated from animal and cellular models of lung fibrosis in the current study, significant curative effect of Andro can be expected to improve IPF care.

\section{Conclusions}

In conclusion, our results confirmed that Andro attenuates BLM-induced pulmonary fibrosis in rats by inhibiting NLRP3 inflammasome activation and EMT in alveolar epithelial cells through AKT/mTOR signaling pathway, 
indicating a pharmacological tool for the therapy of IPF.

\section{Acknowledgments}

We thank Dr. Keng-Leong Ang for the language editing of this work.

Funding: This research was financially supported by the National Natural Science Foundation of China (grant 81703792).

\section{Footnote}

Reporting Checklist: The authors have completed the ARRIVE reporting checklist. Available at http://dx.doi. org/10.21037/atm-20-7973

Data Sharing Statement: Available at http://dx.doi. org/10.21037/atm-20-7973

Conflicts of Interest: All authors have completed the ICMJE uniform disclosure form (available at http://dx.doi. org/10.21037/atm-20-7973). The authors have no conflicts of interest to declare.

Ethical Statement: The authors are accountable for all aspects of the work in ensuring that questions related to the accuracy or integrity of any part of the work are appropriately investigated and resolved. Experiments were performed under a project license (No. 2017-346) granted by the Animal Care and Use Committee of the First Affiliated Hospital of Guangzhou Medical University, in compliance with institutional guidelines for the care and use of animals.

Open Access Statement: This is an Open Access article distributed in accordance with the Creative Commons Attribution-NonCommercial-NoDerivs 4.0 International License (CC BY-NC-ND 4.0), which permits the noncommercial replication and distribution of the article with the strict proviso that no changes or edits are made and the original work is properly cited (including links to both the formal publication through the relevant DOI and the license). See: https://creativecommons.org/ licenses/by-nc-nd/4.0/.

\section{References}

1. King TE Jr, Pardo A, Selman M. Idiopathic pulmonary fibrosis. Lancet 2011;378:1949-61.

2. Raghu G, Collard HR, Egan JJ, et al. An official ATS/ ERS/JRS/ALAT statement: idiopathic pulmonary fibrosis: evidence-based guidelines for diagnosis and management. Am J Respir Crit Care Med 2011;183:788-824.

3. Spagnolo P, Sverzellati N, Rossi G, et al. Idiopathic pulmonary fibrosis: an update. Ann Med 2015;47:15-27.

4. Xiang J, Cheng S, Feng T, et al. Neotuberostemonine attenuates bleomycin-induced pulmonary fibrosis by suppressing the recruitment and activation of macrophages. Int Immunopharmacol 2016;36:158-64.

5. Crystal RG, Bitterman PB, Mossman B, et al. Future research directions in idiopathic pulmonary fibrosis: summary of a National Heart, Lung, and Blood Institute working group. Am J Respir Crit Care Med 2002;166:236-46.

6. Song N, Liu J, Shaheen S, et al. Vagotomy attenuates bleomycin-induced pulmonary fibrosis in mice. Sci Rep 2015;5:13419.

7. B Moore B, Lawson WE, Oury TD, et al. Animal models of fibrotic lung disease. Am J Respir Cell Mol Biol 2013;49:167-79.

8. Moore BB, Hogaboam CM. Murine models of pulmonary fibrosis. Am J Physiol Lung Cell Mol Physiol 2008;294:L152-60.

9. Ma Y, Wang M, Li N, et al. Bleomycin-induced nuclear factor-kappaB activation in human bronchial epithelial cells involves the phosphorylation of glycogen synthase kinase 3beta. Toxicol Lett 2009;187:194-200.

10. Tian R, Zhu Y, Yao J, et al. NLRP3 participates in the regulation of EMT in bleomycin-induced pulmonary fibrosis. Exp Cell Res 2017;357:328-34.

11. Chen YL, Zhang X, Bai J, et al. Sorafenib ameliorates bleomycin-induced pulmonary fibrosis: potential roles in the inhibition of epithelial-mesenchymal transition and fibroblast activation. Cell Death Dis 2013;4:e665.

12. Bartis D, Mise N, Mahida RY, et al. Epithelialmesenchymal transition in lung development and disease: does it exist and is it important? Thorax 2014;69:760-5.

13. Kim KK, Kugler MC, Wolters PJ, et al. Alveolar epithelial cell mesenchymal transition develops in vivo during pulmonary fibrosis and is regulated by the extracellular matrix. Proc Natl Acad Sci U S A 2006;103:13180-5.

14. Willis BC, Liebler JM, Luby-Phelps K, et al. Induction of epithelial-mesenchymal transition in alveolar epithelial cells by transforming growth factor-beta1: potential role in idiopathic pulmonary fibrosis. Am J Pathol 2005;166:1321-32. 
15. Tanjore H, Xu XC, Polosukhin VV, et al. Contribution of epithelial-derived fibroblasts to bleomycin-induced lung fibrosis. Am J Respir Crit Care Med 2009;180:657-65.

16. Stout-Delgado HW, Cho SJ, Chu SG, et al. AgeDependent Susceptibility to Pulmonary Fibrosis Is Associated with NLRP3 Inflammasome Activation. Am J Respir Cell Mol Biol 2016;55:252-63.

17. Cho SJ, Moon JS, Nikahira K, et al. GLUT1-dependent glycolysis regulates exacerbation of fibrosis via AIM2 inflammasome activation. Thorax 2020;75:227-36.

18. Meng Y, Pan M, Zheng B, et al. Autophagy Attenuates Angiotensin II-Induced Pulmonary Fibrosis by Inhibiting Redox Imbalance-Mediated NOD-Like Receptor Family Pyrin Domain Containing 3 Inflammasome Activation. Antioxid Redox Signal 2019;30:520-41.

19. Lasithiotaki I, Giannarakis I, Tsitoura E, et al. NLRP3 inflammasome expression in idiopathic pulmonary fibrosis and rheumatoid lung. Eur Respir J 2016;47:910-8.

20. Song C, He L, Zhang J, et al. Fluorofenidone attenuates pulmonary inflammation and fibrosis via inhibiting the activation of NALP3 inflammasome and IL-1beta/IL1R1/MyD88/NF-kappaB pathway. J Cell Mol Med 2016;20:2064-77.

21. Sheeja K, Kuttan G. Activation of cytotoxic T lymphocyte responses and attenuation of tumor growth in vivo by Andrographis paniculata extract and andrographolide. Immunopharmacol Immunotoxicol 2007;29:81-93.

22. Ji X, Li C, Ou Y, et al. Andrographolide ameliorates diabetic nephropathy by attenuating hyperglycemiamediated renal oxidative stress and inflammation via Akt/NF-kappaB pathway. Mol Cell Endocrinol 2016;437:268-79.

23. Xia H, Xue J, Xu H, et al. Andrographolide antagonizes the cigarette smoke-induced epithelial-mesenchymal transition and pulmonary dysfunction through antiinflammatory inhibiting HOTAIR. Toxicology 2019;422:84-94.

24. Peng S, Gao J, Liu W, et al. Andrographolide ameliorates OVA-induced lung injury in mice by suppressing ROSmediated NF-kappaB signaling and NLRP3 inflammasome activation. Oncotarget 2016;7:80262-74.

25. Zhu T, Zhang W, Xiao M, et al. Protective role of andrographolide in bleomycin-induced pulmonary fibrosis in mice. Int J Mol Sci 2013;14:23581-96.

26. Yin JN, Li YN, Gao Y, et al. Andrographolide plays an important role in bleomycin-induced pulmonary fibrosis treatment. Int J Clin Exp Med 2015;8:12374-81.

27. Li J, Feng M, Sun R, et al. Andrographolide ameliorates bleomycin-induced pulmonary fi brosis by suppressing cell proliferation and myofibroblast differentiation of fibroblasts via the TGF-beta1-mediated Smaddependent and -independent pathways. Toxicol Lett 2020;321:103-13.

28. Karkale S, Khurana A, Saifi MA, et al. Andrographolide ameliorates silica induced pulmonary fibrosis. Int Immunopharmacol 2018;62:191-202.

29. Gao J, Peng S, Shan X, et al. Inhibition of AIM2 inflammasome-mediated pyroptosis by Andrographolide contributes to amelioration of radiation-induced lung inflammation and fibrosis. Cell Death Dis 2019;10:957.

30. Li J, Liu J, Yue W, et al. Andrographolide attenuates epithelial-mesenchymal transition induced by TGFbeta1 in alveolar epithelial cells. J Cell Mol Med 2020;24:10501-11.

31. Ashcroft T, Simpson JM, Timbrell V. Simple method of estimating severity of pulmonary fibrosis on a numerical scale. J Clin Pathol 1988;41:467-70.

32. Sakai N, Tager AM. Fibrosis of two: Epithelial cellfibroblast interactions in pulmonary fibrosis. Biochim Biophys Acta 2013;1832:911-21.

33. Hsu HS, Liu CC, Lin JH, et al. Involvement of ER stress, PI3K/AKT activation, and lung fibroblast proliferation in bleomycin-induced pulmonary fibrosis. Sci Rep 2017;7:14272.

34. Guan R, Wang X, Zhao X, et al. Emodin ameliorates bleomycin-induced pulmonary fibrosis in rats by suppressing epithelial-mesenchymal transition and fibroblast activation. Sci Rep 2016;6:35696.

35. Pardo A, Selman M. Molecular mechanisms of pulmonary fibrosis. Front Biosci 2002;7:d1743-61.

36. Ikegami T, Zhang Y, Matsuzaki Y. Liver fibrosis: possible involvement of EMT. Cells Tissues Organs 2007;185:213-21.

37. Ramsgaard L, Englert JM, Tobolewski J, et al. The role of the receptor for advanced glycation end-products in a murine model of silicosis. PLoS One 2010;5:e9604.

38. He M, Kubo H, Ishizawa K, et al. The role of the receptor for advanced glycation end-products in lung fibrosis. Am J Physiol Lung Cell Mol Physiol 2007;293:L1427-36.

39. Cabrera D, Wree A, Povero D, et al. Andrographolide Ameliorates Inflammation and Fibrogenesis and Attenuates Inflammasome Activation in Experimental Non-Alcoholic Steatohepatitis. Sci Rep 2017;7:3491.

40. Ahmed S, Kwatra M, Ranjan PS, et al. Andrographolide suppresses NLRP3 inflammasome activation in microglia through induction of parkin-mediated mitophagy in in- 
vitro and in-vivo models of Parkinson disease. Brain Behav Immun 2021;91:142-58.

41. Zhuo X, Wu Y, Yang Y, et al. Knockdown of LSD1 meliorates Ox-LDL-stimulated NLRP3 activation and inflammation by promoting autophagy via SESN2mesiated PI3K/Akt/mTOR signaling pathway. Life Sci 2019;233:116696.

Cite this article as: Li J, Yang X, Yang P, Xu K, Peng X, Cai W, Zhao S, Hu L, Li Z, Cui F, Wang W, Peng G, Xu X, He J, Liu J. Andrographolide alleviates bleomycin-induced NLRP3 inflammasome activation and epithelial-mesenchymal transition in lung epithelial cells by suppressing AKT/mTOR signaling pathway. Ann Transl Med 2021;9(9):764. doi: 10.21037/atm-20-7973
42. Saito S, Zhuang Y, Shan B, et al. Tubastatin ameliorates pulmonary fibrosis by targeting the TGFbeta-PI3K-Akt pathway. PLoS One 2017;12:e0186615.

43. Karimi Roshan M, Soltani A, Soleimani A, et al. Role of AKT and mTOR signaling pathways in the induction of epithelial-mesenchymal transition (EMT) process. Biochimie 2019;165:229-34. 